\title{
INFLUÊNCIA DO TIPO E DA CONCENTRAÇÃO DE QUEBRADORES DE GÉIS NO PROCESSO DE FRATURAMENTO HIDRÁULICO
}

\author{
M. A. CAVALCANTE; S. G. CHAVES; F. P. FAGUNDES e K. R. S. FAGUNDES* \\ Universidade Federal Rural do Semiárido, Engenharia de Petróleo \\ keilaregina@ufersa.edu.br*
}

Submetido 10/10/2016 - Aceito 12/12/2016

DOI: $10.15628 /$ holos.2017.5196

\section{RESUMO}

Umas das técnicas mais utilizadas até hoje para auxiliar o aumento da produção de óleo e gás de reservatórios subterrâneos é o fraturamento hidráulico. Nesta técnica, um fluido viscoso é bombeado para o fundo do poço a uma pressão suficientemente elevada, visando à criação de uma fratura de alta condutividade na formação de interesse. $O$ fluido de fraturamento (gel de fraturamento) transporta sólidos responsáveis pela sustentação da fratura (areia, bauxita ou cerâmica) os quais asseguram a existência de um canal efetivo e permanentemente aberto ao fluxo de hidrocarbonetos após o fim do bombeio. Normalmente, as soluções poliméricas são extensivamente utilizadas na preparação desses géis. Uma característica importante destes é ser facilmente quebrado no fim da operação, para que os seus resíduos não venham a tamponar os poros recém-criados. De acordo com a literatura, um gel completamente quebrado (Linear ou reticulado) é
\end{abstract}

aquele cuja viscosidade é aproximadamente menor que $10 \mathrm{cP}$. Com isso, o objetivo deste trabalho consiste em avaliar a eficiência de diferentes tipos e concentrações (5\%, 15\%, 30\%) dos "quebradores de gel" (Ácido Clorídrico, Persulfato de amônio e cloreto de cálcio) em função das propriedades reológicas do fluido de fraturamento. De acordo com os resultados obtidos podemos afirmar que o $\mathrm{HCl} 15 \%$ utilizado como quebrador, apesar de promover a quebra completa do gel acaba gerando alguns transtornos associados ao reservatório, podendo promover obstruções e acarretando um aumento no dano a formação. O cloreto de cálcio funcionou mais como um agente de precipitação do que propriamente como um quebrador. Assim, o persulfato de amônio, foi entre os quebradores estudados, o mais eficiente no processo de faturamento hidráulico tendo em vista os menores valores de viscosidade.

PALAVRAS-CHAVE: Fraturamento hidráulico, géis de fraturamento, quebrador de géis.

\section{INFLUENCE OF TYPE AND CONCENTRATION OF GEL BREAKERS IN HYDRAULIC FRACTURING PROCESS}

\begin{abstract}
Nowadays, one of the most important techniques used to promote the increase of production of oil and gas in reservoirs is the hydraulic fracturing. This technique, a viscous fluid is pumped to borehole under high pressure, aiming the creating of fracture with high conductivity about zone of interest. The fracturing fluid (fracturing gel) transports solids responsible to suspend the fracture zone (sand, bauxite or ceramic material), which are responsible to promote an effective canal and permanently opened about the flux of hydrocarbons after to finish the pumping. Normally, polymeric solutions are extensively used to prepare fracturing gels. These hydrogels present high viscosity in low concentrations of polymer. One important characteristic refers to the gel break process after the fracturing process due to possibility of obstruction by
\end{abstract}

residues generated during this process. A gel is considered totally broken (linear or crosslinked) when its viscosity reaches values below $10 \mathrm{cP}$. In this context, the main objective of this work is evaluate the performance of different kind (hydrochloric acid, ammonium persulfate and calcium chloride) and concentrations of breakers $(5 \%, 15 \%, 30 \%)$ as function of rheological properties of fracturing fluid. According to results obtained, it can be proved that hydrochloric acid $(\mathrm{HCl})$ $15 \%$ used as breaker is responsible to generate obstructions and to promote formation damage. The breaker calcium chloride $\left(\mathrm{CaCl}_{2}\right)$ was more efficient as precipitation agent than breaker. Amonium persulfate presented the higher values of breaking compared to others breakers, in view of the values of apparent viscosity less than 10 cP. 


\section{INTRODUÇÃO}

A técnica de estimulação através do fraturamento hidráulico tem como objetivo aumentar a produtividade de poços de petróleo ou gás. Nesta técnica, um fluido viscoso (normalmente nãoNewtoniano) é bombeado para o fundo do poço a uma pressão suficientemente elevada, visando à criação de uma fratura de alta condutividade na formação de interesse. A fratura inicia-se junto à parede do poço e propaga-se em direção ao interior da formação à medida que o fluido é injetado. O fluido de fraturamento transporta sólidos responsáveis pela sustentação da fratura (areia, bauxita ou cerâmica), os quais asseguram a existência de um canal efetivo e permanentemente aberto ao fluxo de hidrocarbonetos após o fim do bombeio (GROTHE, 2000; KING, 2012)).

De acordo com Oliveira (2012), existem diversas aplicações para o fraturamento hidráulico, entre eles, algumas como: Aumento da área de drenagem ou o contato entre a formação e o poço; Aumento da vazão de hidrocarbonetos produzidos de reservatórios de baixa permeabilidade ou de poços danificados; Conexão de fraturas naturais; Redução da queda de pressão ao redor do poço a fim de minimizar a produção de areia ou minimizar problemas como a deposição de asfaltenos e parafinas. Desta forma, o fraturamento poderá agir permitindo, facilitando ou ainda acelerando a produção de hidrocarbonetos presentes no reservatório.

Duas etapas básicas estão envolvidas nesta técnica, a primeira é a injeção do fluido e a outra é a colocação de um agente propante com o objetivo de manter a fratura quando a aplicação da pressão for aliviada. Para a retomada da produção, a quebra do gel é necessária para manter a alta condutividade da fratura. Este processo é feito pela injeção de um quebrador, que reage com as cadeias do polissacarídeo promovendo sua degradação ao longo do tempo (PEREIRA et al, 2011; BARATI et al, 2009; VENGOSH et al, 2014).

Porém, os fluidos de fraturamento podem causar dano à formação e/ou à condutividade da fratura devido à presença em meio poroso de: emulsões, precipitados, argilas ou outros finos, fluido com alta viscosidade ou resíduos insolúveis. O dano causado por fluidos aquosos viscosos resulta de dois mecanismos: Resíduos insolúveis contidos inicialmente no fluido de fraturamento ou que foram formados durante a degradação do fluido; Alta concentração de polímero na face da fratura devido à perda de fluido para a formação (STRINGFELLOW et al,2014).

Nesse contexto, as soluções poliméricas são extensivamente utilizadas na preparação de géis de fraturamento hidráulico. Esses hidrogéis apresentam elevada viscosidade em baixas concentrações de polímero, devido à presença de ligações cruzadas entre as cadeias poliméricas. Além de possuir essa elevada viscosidade, para garantir uma boa capacidade de suspensão e transporte do agente propante ao longo da fratura durante o processo de injeção, o fluido de fraturamento deve possuir a característica de ser facilmente quebrado no fim da operação, para que os seus resíduos não venham a tamponar os poros recém-criados, mantendo, portanto, a elevada condutividade da fratura durante a fase de produção. No entanto, o uso de fluidos poliméricos resulta em uma quantidade de resíduos que permanece na fratura após o tratamento. 
O processo de quebra de gel é um ponto importante a ser estudado, pois o mesmo está intrinsicamente associado ao dano a formação. O polímero presente em fluidos de fraturamento convencionais pode reduzir a produção do poço devido à formação de reboco nas faces da fratura e de resíduos dentro do pacote do agente de sustentação. A quebra do gel possibilita o retorno rápido do fluido para a superfície, facilitando a limpeza da fratura, no caso da aplicação dos géis em fraturamento hidráulico. Diante desse cenário constitui um desafio chave o entendimento do efeito que cada tipo de quebrador utilizado exerce sobre as propriedades reológicas e estruturais dos géis de fraturamento, de forma a minimizar o dano à formação. Logo, o objetivo deste trabalho consiste em avaliar diferentes concentrações dos "quebradores de gel" (Ácido Clorídrico, Persulfato de amônia e cloreto de cálcio) em função das propriedades reológicas do fluido de fraturamento utilizado (Hidrogel à base de hidroxipropilguar).

\section{MATERIAIS E MÉTODOS}

\subsection{Materiais}

Os materiais utilizados na preparação dos géis poliméricos foram: hidroxipropilguar (HPG) e o ácido bórico $\left(\mathrm{H}_{3} \mathrm{BO}_{3}\right)$ ambos doados pela PETROBRAS. O cloreto de potássio (PROQUÍMIOS), hidróxido de sódio (IMPEX) foram utilizados de grau analítico. Da mesma forma, os quebradores ácido clorídrico (PROQUÍMIOS), persulfato de amônia (LABSYNTH) e cloreto de cálcio (PROQUÍMIOS).

\subsection{Preparação dos géis reticulados}

Os géis poliméricos (Tabela 1) foram preparados em um agitador Hamilton Beach (1 bbl/equivalente $-350,5 \mathrm{~mL}$ ), adicionando sequencialmente todos os aditivos. Os aditivos foram incorporados ao fluido em intervalos de 10 minutos e, posteriormente o processo de reticulação foi concluído com a adição de $1 \mathrm{ml}$ de hidróxido de sódio (8 M).

Tabela 1: Formulação do hidrogel base

\begin{tabular}{c|c}
\hline Aditivo & Concentração \\
\hline $\mathrm{HPG}(\mathrm{lb} / \mathrm{bbl})$ & 2 \\
$\mathrm{KCl}(\mathrm{lb} / \mathrm{bbl})$ & 10 \\
$\mathrm{H}_{3} \mathrm{BO}_{3}(\mathrm{lb} / \mathrm{bbl})$ & 1 \\
$\mathrm{NaOH} 8 \mathrm{M}(\mathrm{ml})$ & 1 \\
\hline
\end{tabular}

\subsection{Processo de Quebra dos géis reticulados}

Os géis foram submetidos à diferentes temperaturas $\left(50^{\circ} \mathrm{C}, 75^{\circ} \mathrm{C}\right.$ e $\left.100^{\circ} \mathrm{C}\right)$ em presença de diferentes concentrações $(5 \%, 15 \%$ e $30 \%)$ de cada quebrador (ácido clorídrico $(\mathrm{HCl})$, persulfato de amônia $\left(\left(\mathrm{NH}_{4}\right)_{2} \mathrm{~S}_{2} \mathrm{O}_{8}\right)$ e cloreto de cálcio $\left.\left(\mathrm{CaCl}_{2}\right)\right)$ em função da cinética do tempo. A eficiência de quebra foi avaliada com base na massa restante de polímero (g) e na redução da viscosidade para valores próximos de $10 \mathrm{cP}$. 


\subsection{Análise de Perda de Massa}

A avaliação de perda de massa dos géis foi realizada utilizando um aparato contendo um funil Büchner acoplado a um becker, onde os géis juntamente com os diferentes quebradores foram colocados e submetidos a diferentes temperaturas durante 3 horas, onde com base na redução da perda de massa na fase superior, foi possível calcular o percentual de resíduo pós-quebra (Equação 1). Diferentes temperaturas, tempo e concentrações do quebrador foram avaliados.

$$
\Delta P=\frac{S_{g}-S_{s g}}{S_{g}} \times 100
$$

Onde: $\Delta \mathrm{P}$ indica o diferencial da pesagem do sistema inicial e final, o $\mathrm{S}_{\mathrm{g}}$ e $\mathrm{S}_{\mathrm{sg}}$ representa peso inicial e final do sistema $(\mathrm{g})$, respectivamente.

\subsection{Determinação do grau de turbidez das amostras}

De acordo com a literatura, a turbidez representa a medida da dificuldade de um feixe de luz atravessar uma certa quantidade da amostra selecionada. Essa medição é feita com o turbidímetro, que compara o espalhamento de um feixe de luz ao passar por uma suspensão padrão. Quanto maior o espalhamento, maior será a turbidez da amostra. Dessa forma, o teste de turbidez foi realizado em um equipamento de turbidímetro, da marca Policontrol, com o intuito de comprovar a quebra do polímero de HPG no meio após sua quebra. Foram adicionados $5 \mathrm{~mL}$ de álcool em cada uma das amostras para precipitar o polímero no meio. Em seguida, o grau de turbidez foi medido com base em uma curva de calibração de concentração previamente conhecida (NTU).

\subsection{Determinação da viscosidade aparente dos géis pós-quebra}

As viscosidades aparentes dos géis submetidos a diferentes tipos e concentração de quebradores e temperatura foram determinadas em um viscosímetro rotativo da Fann Instrument Company, modelo 35 A, combinação de R1-B1 e mola de torção F1. A análise reológica de cada gel foi realizada na rotação de $600 \mathrm{rpm}$ e, posteriormente, a viscosidade aparente (VA) foram determinadas, conforme a norma API.

\subsection{Parâmetros Analisados}

Foi utilizado um planejamento fatorial $3^{3}$, com três níveis e três variáveis (mínimos (-1), intermediários (0) e máximos (+1)), como mostra a Tabela 2 para avaliar a interação dos parâmetros utilizados (tipo de quebrador, concentração de quebrador e temperatura), tendo como variáveis de resposta: Perda de massa, turbidez e viscosidade aparente.

Tabela 2: Intervalo de análise dos parâmetros avaliados

\begin{tabular}{l|c|c|c}
\hline \multicolumn{1}{c}{ Parâmetro } & Mínimo (-1) & Intermediário (0) & Máximo (+1) \\
\hline Temperatura $\left({ }^{\circ} \mathrm{C}\right)$ & 50 & 75 & 100 \\
Concentração de & 5 & 15 & 30 \\
Quebrador (\%) & $\mathrm{HCl}$ & $\left(\mathrm{NH}_{4}\right)_{2} \mathrm{~S}_{2} \mathrm{O}_{8}$ & $\mathrm{CaCl}_{2}$ \\
Tipo de Quebrador & & &
\end{tabular}


Para tratamento dos dados, o programa estatístico Statistica Software versão 7.0 foi empregado para obtenção dos efeitos, diagrama de pareto e as superfícies de respostas.

\section{RESULTADOS E DISCUSSÃO}

A ação de diferentes quebradores foi avaliada em função da redução de massa polimérica dos géis à base de HPG quando submetido à diferentes temperaturas. As propriedades reológicas foram determinadas a fim de classificar o gel em totalmente quebrado, parcialmente quebrado e não quebrado. Em paralelo, testes reológicos e de tubidez foram determinados com o intuito de associar a viscosidade e a turbidez do gel pós-quebra à eficiência de quebra do sistema, tendo em vista que, de acordo com a literatura, para ser considerado um gel totalmente quebrado sua viscosidade não pode ultrapassar o valor de 10 cP (ZAMORA et al., 2010).

\subsection{1. Percentual de perda de massa dos géis reticulados}

Inicialmente, foi realizado um estudo através dos gráficos de Pareto (Figura 1) para se observar a sensibilidade do percentual de perda de massa em relação aos intervalos de parâmetros avaliados.

Um valor positivo ao lado da barra indica que, quanto maior o parâmetro, maior o percentual de perda de massa. Por outro lado, um valor negativo indica que com um aumento do parâmetro, ocorre uma diminuição no percentual de perda de massa. Os efeitos cujos retângulos estiverem à direita da linha divisória $(p=0,05)$ são estatisticamente significativos ao nível de $95 \%$ de confiança sobre a resposta.

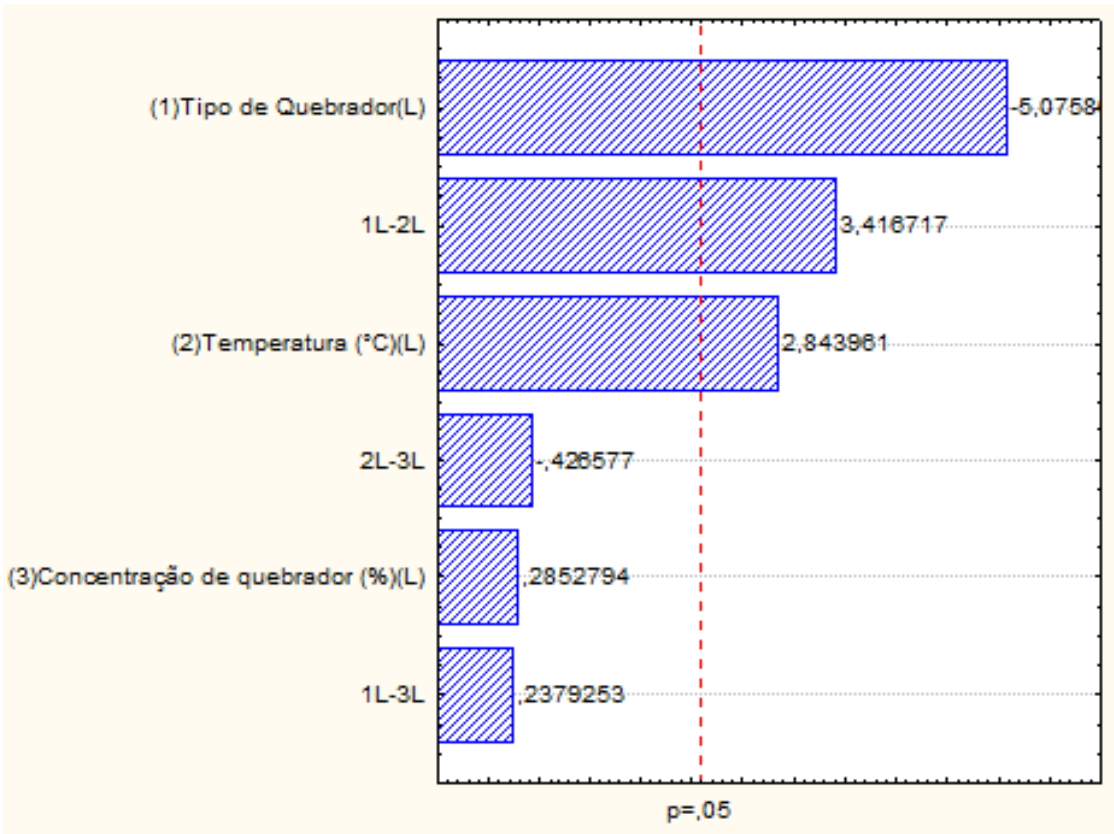

Figura 1: Diagrama de Pareto - Percentual de perda de massa 
Os efeitos principais estatisticamente relevantes são:

- $\quad$ Tipo de quebrador: o efeito foi significante, porém negativo, logo espera-se um incremento no percentual de perda de massa quando o $\mathrm{HCl}(-1)$ for utilizado.

- Temperatura: o efeito foi significante com sinal positivo, logo para o nível máximo de temperatura utilizado $\left(100^{\circ} \mathrm{C}\right)$ tem-se um aumento no percentual de perda de massa.

A Figura 2 apresenta o percentual de perda de massa em função do tempo para os géis com diferentes concentrações de $\mathrm{HCl}$ submetidos as diferentes temperaturas $\left(50^{\circ} \mathrm{C}, 75^{\circ} \mathrm{C}\right.$ e $\left.100^{\circ} \mathrm{C}\right)$.

(I)

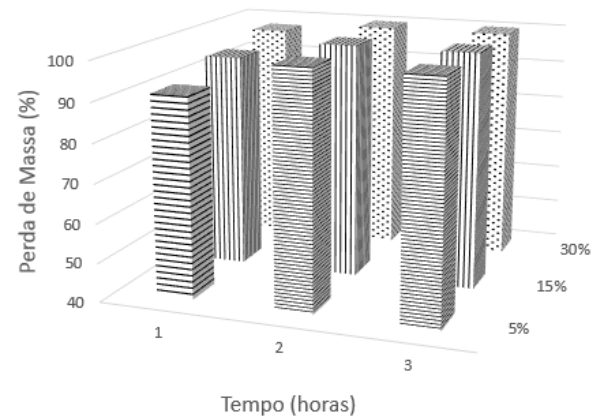

(II)

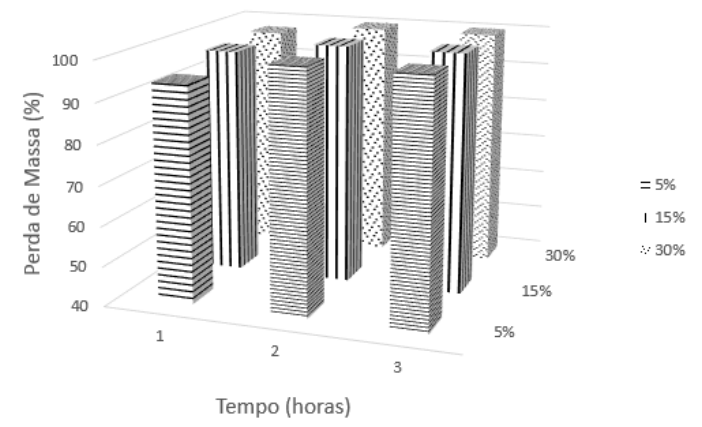

(III)

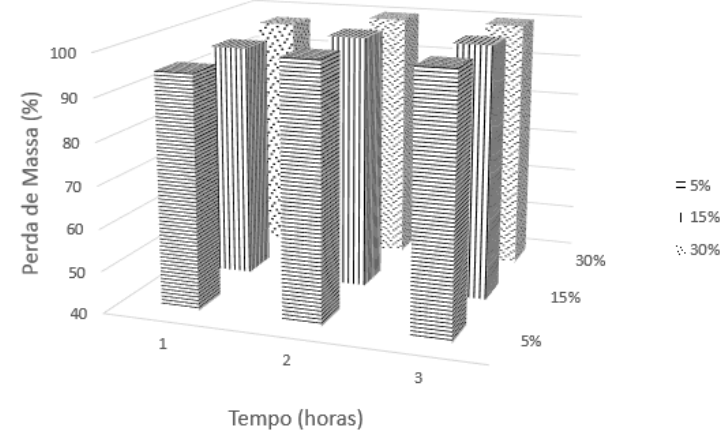

Figura 2: Percentual de perda de massa (\%) em função do tempo para as diferentes concentrações de $\mathrm{HCl}$ nas temperaturas de $50^{\circ} \mathrm{C}$ (I), $75^{\circ} \mathrm{C}$ (II) e $100^{\circ} \mathrm{C}$ (III).

De acordo com a Figura 2, percebe-se que houve uma redução na massa polimérica de $100 \%$ já nas primeiras horas em todas as concentrações de $\mathrm{HCl} 15 \%$ e em todas as temperaturas. Esse efeito pode ser explicado devido ao alto poder de dissociação em íons $\mathrm{H}^{+}$e $\mathrm{Cl}^{-}(\mathrm{pKa}<1,0), \mathrm{o}$ que certamente foi responsável por quebrar mais rapidamente o gel de HPG. Por outro lado, essa rápida perda de massa residual pode gerar alguns transtornos associados ao reservatório, causado, possivelmente, pela reação desse ácido com constituintes da formação promovendo a obstrução sob a forma de incrustações.

A Figura 3 apresenta o percentual de perda de massa em função do tempo para os géis com diferentes concentrações de Persulfato de amônia submetidos a diferentes temperaturas $\left(50^{\circ} \mathrm{C}, 75^{\circ} \mathrm{C}\right.$ e $\left.100^{\circ} \mathrm{C}\right)$. 
(1)

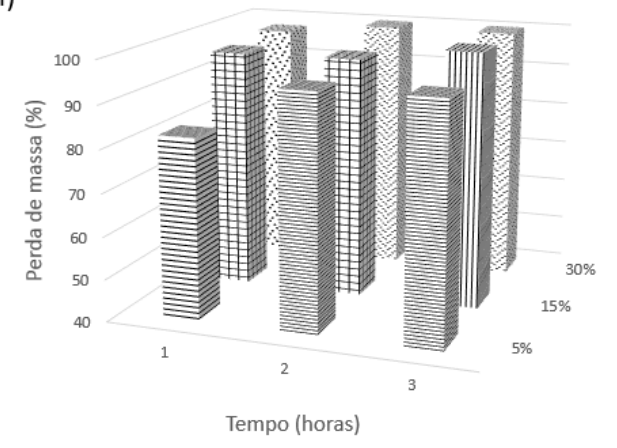

(II)

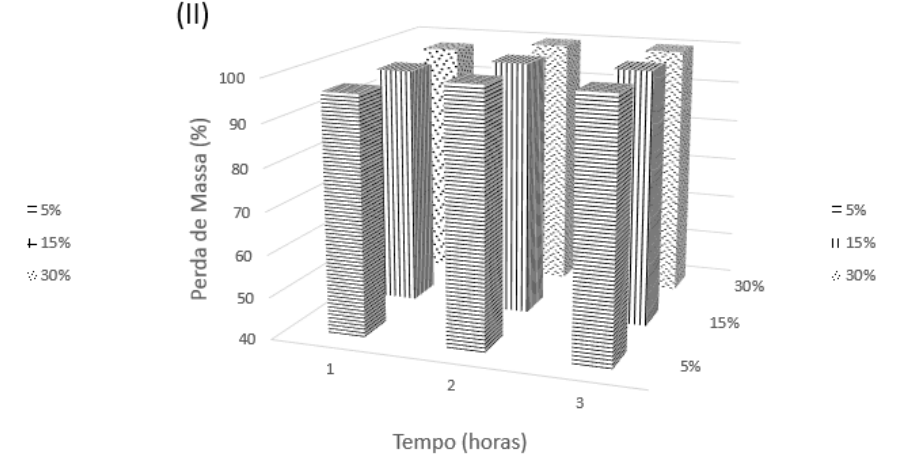

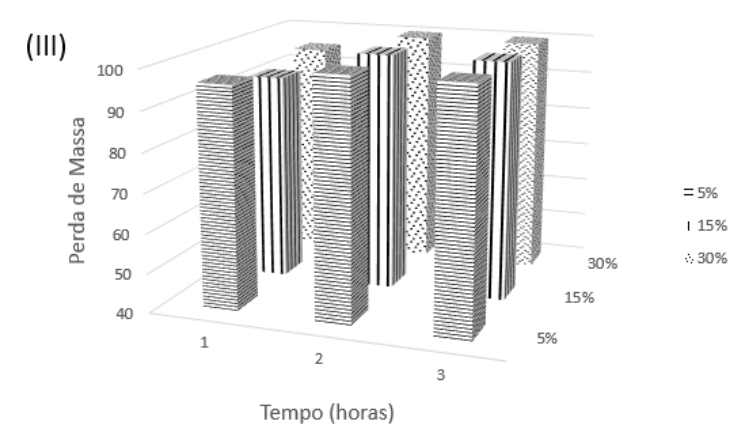

Figura 3: Percentual de perda de massa (\%) em função do tempo para as diferentes concentrações de Persultato de amônia nas temperaturas de $50^{\circ} \mathrm{C}(\mathrm{I}), 75^{\circ} \mathrm{C}$ (II) e $100^{\circ} \mathrm{C}$ (III).

A associação do quebrador com a temperatura do meio representa um importante aspecto para avaliar sua eficiência quanto ao percentual de perda de massa, conforme pode ser observado na Figura 3. A maioria das propriedades químicas do persulfato de amônio são devidas ao ânion persulfato $\left(\mathrm{S}_{2} \mathrm{O}_{8}\right)^{2-}$. O ânion persulfato, que possui um potencial de redução de $2,01 \mathrm{~V}$, é um agente oxidante muito forte e capaz de degradar uma série de compostos orgânicos (CARVALHO, 2011), inclusive quebrar grande parte das estruturas reticuladas do HPG, promovendo assim a perda de massa. Essa reação produz amônia e sais sulfurosos que retornam a superfície junto com a água, o que pôde ser observado em virtude da redução do pH após a quebra dos géis reticulados.

A Figura 4 apresenta o percentual de perda de massa em função do tempo para os géis com diferentes concentrações de cloreto de cálcio submetidos as diferentes temperaturas $\left(50^{\circ} \mathrm{C}\right.$, $75^{\circ} \mathrm{C}$ e $\left.100^{\circ} \mathrm{C}\right)$. 
(I)

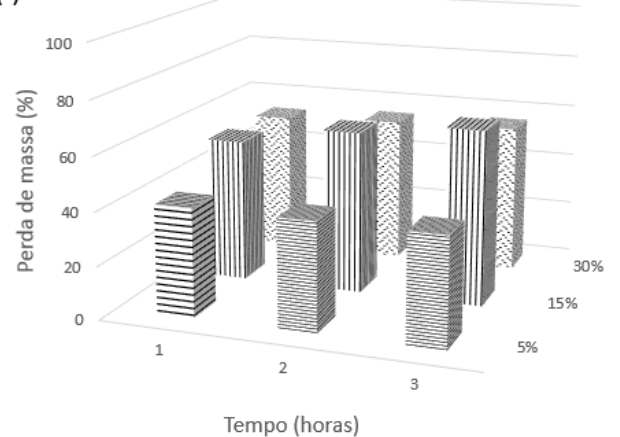

(II)

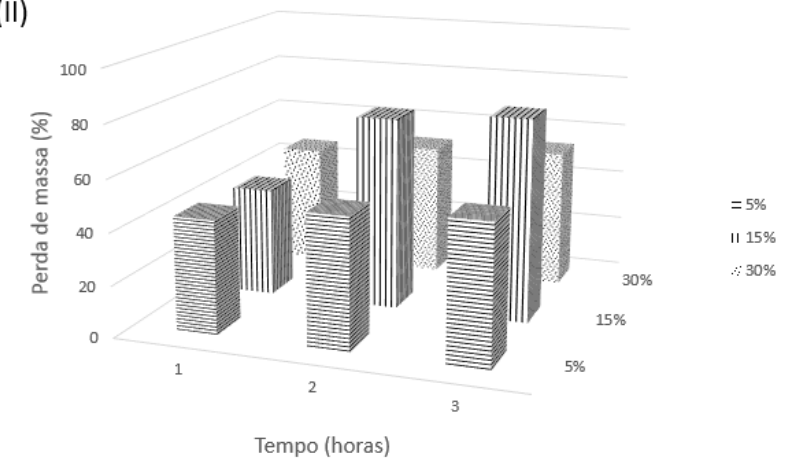

(III)

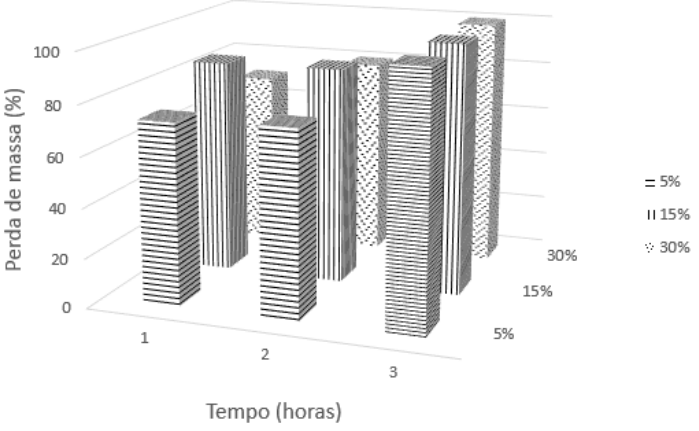

Figura 4: Percentual de perda de massa (\%) em função do tempo para as diferentes concentrações de $\mathrm{CaCl}_{2}$ nas temperaturas de $50^{\circ} \mathrm{C}$ (I), $75^{\circ} \mathrm{C}$ (II) e $100^{\circ} \mathrm{C}$ (III)

De acordo com a Figura 4, não houve redução significativa da massa residual usando diferentes concentrações do quebrador cloreto de cálcio em associação com as temperaturas $50^{\circ} \mathrm{C}$ (I) e $75^{\circ} \mathrm{C}$ (II). Em contrapartida na Figura 4 (III) é verificado um melhor desempenho desse sistema, mostrando um aumento na perda de massa.

O cloreto de cálcio não obteve um bom desempenho como quebrador provavelmente por ser uma substância fortemente higroscópica, ou seja, não degrada o polímero mas interage com a fração aquosa do gel de fraturamento.

\subsection{Comportamento reológico pós-quebra dos géis}

A Figura 5 mostra o diagrama de Pareto para se observar a sensibilidade das propriedades reológicas em relação aos intervalos dos parâmetros avaliados. 


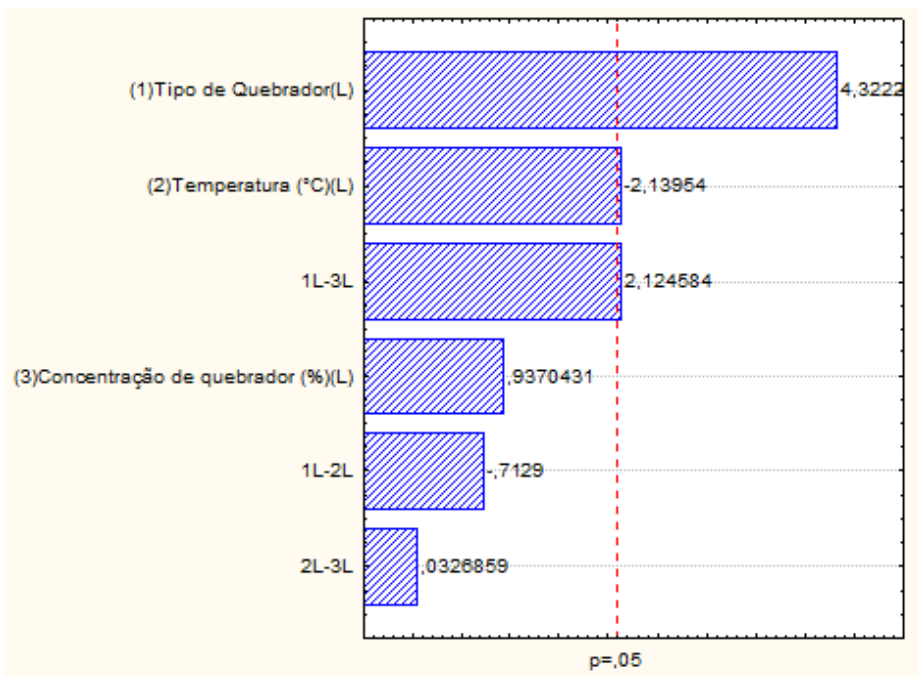

Figura 5: Diagrama de Pareto - Viscosidade Aparente

De acordo com a Figura 5, os principais efeitos estatisticamente relevantes são:

- Tipo de quebrador: o efeito foi significante com sinal positivo, logo espera-se um aumento na viscosidade aparente quando o $\mathrm{CaCl}_{2}(+1)$ for utilizado, esse efeito se deve a precipitação do polímero pela presença do íon $\mathrm{Ca}^{2+}$.

- Temperatura: o efeito foi significante, porém negativo, logo, como era esperado, para maiores temperaturas $\left(100^{\circ} \mathrm{C}\right)$ tem-se uma redução da viscosidade aparente dos géis, esse efeito se deve ao aumento da energia interna do sistema, reduzindo o atrito entre elas e favorecendo a redução da viscosidade.

A Figura 6 mostra a viscosidade aparente (cP) em função da concentração de quebrador para os sistemas contendo $\mathrm{HCl}(\mathrm{I})$, persulfato de amônia (II) e $\mathrm{CaCl}_{2}$ (III) à temperatura de $50^{\circ} \mathrm{C}$, $75^{\circ} \mathrm{C}$ e $100^{\circ} \mathrm{C}$. 

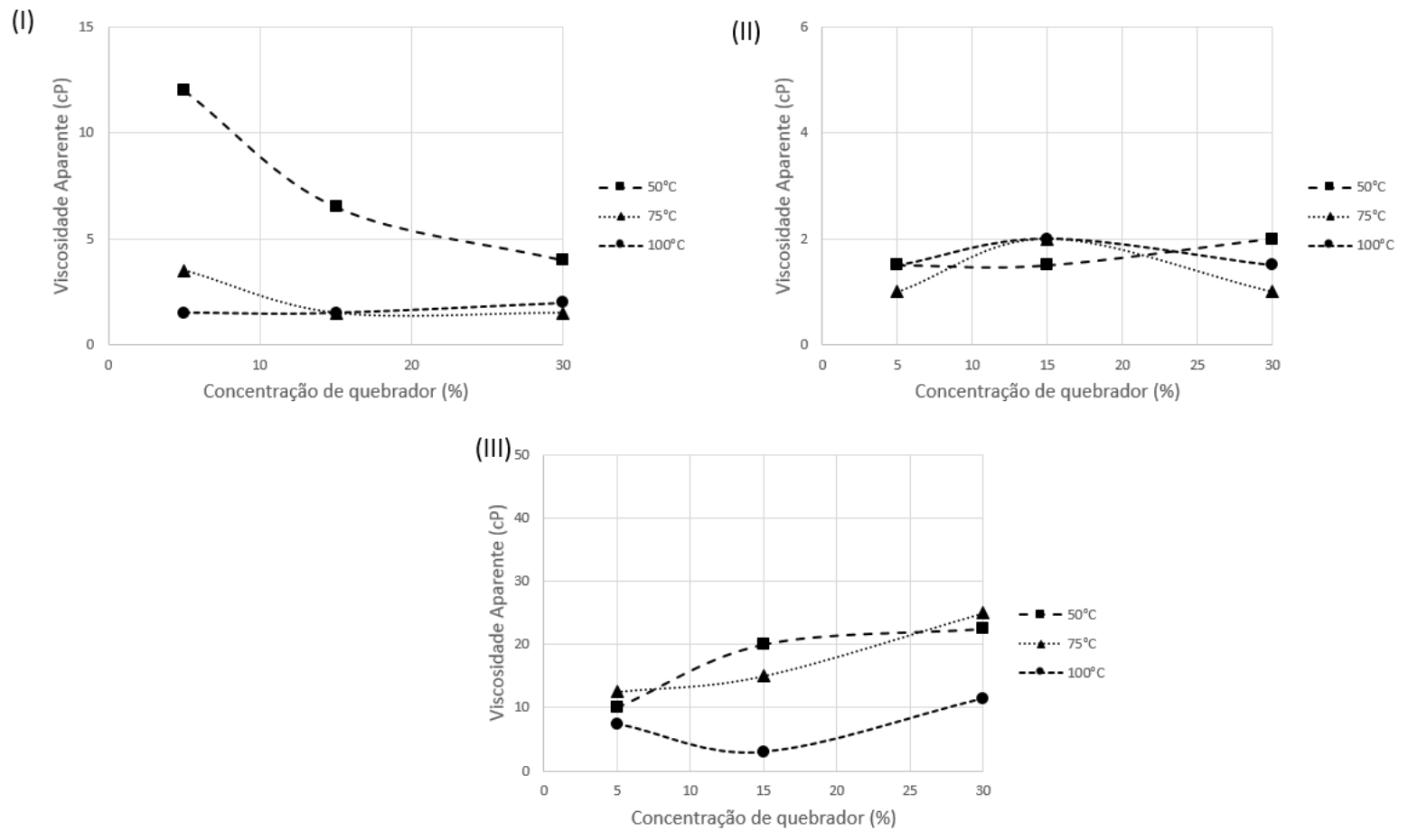

Figura 6: Viscosidade Aparente (CP) em função da concentração do quebrador de (I) HCl, (II) persulfato de amônio e (III) cloreto de cálcio

De acordo com Zamora e colaboradores (2010), a quebra completa dos géis, refere-se a um fenômeno no qual a viscosidade de um gel é reduzida até tal nível que o gel possa ser expelido da formação pelo fluxo de fluidos formados ou que possa ser recuperado por operação de pistoneio (swabbing). Em laboratório, um gel linear completamente quebrado é aquele cuja viscosidade é aproximadamente $10 \mathrm{cP}$ ou menos, conforme medida em viscosímetro modelo 35 Fann equipado com rotor R1B1.

De acordo com a Figura 6 percebe-se três comportamentos distintos para cada quebrador utilizado. A Figura 6 (I) apresenta uma redução da viscosidade em todas as temperaturas utilizadas, onde é possível observar que todos os géis foram completamente quebrados (viscosidade aparente menor que 10cP), esse efeito se deve ao $\mathrm{HCl} 15 \%$ promover uma forte interação dos íons presentes com os sítios ativos do gel reticulado, causando, portanto, uma redução no volume hidrodinâmico da molécula e assim a redução de viscosidade.

De acordo com a Figura 6 (II) não houve variações significativas de viscosidade aparente com o aumento da concentração de persulfato de amônia e da temperatura na faixa estudada. A concentração com $5 \%$ de perssulfato de amônia foi suficiente para reduzir a viscosidade em limites aceitáveis considerados de "quebra". O persulfato de amônio se reduz a ânion persulfato $\left(\mathrm{S}_{2} \mathrm{O}_{8}\right)^{2-}$, o qual é responsável por produzir "radicais livres" que atacam e degradam o polímero. De acordo com a literatura, existem 18 locais potenciais para uma reação de radicais livres em uma única unidade de repetição de guar (cerca de 66.600 moléculas por sítios oxidáveis), o que torna essa reação um verdadeiro processo catalítico. 
Por último, a Figura 6 (III) mostra um aumento da viscosidade aparente com o aumento da concentração de $\mathrm{CaCl}_{2}$, esse efeito se deve ao fato da presença do íon cálcio, na mistura, ser responsável por promover uma maior interação com as cadeias dos polímeros presentes nos géis, através do aumento das interações hidrofóbicas, dando origem a estruturas mais organizadas e rígidas, ocasionando um incremento na viscosidade aparente, em paralelo, os cátions $\mathrm{Ca}^{2+}$ são responsáveis por promover precipitação do polímero no meio, o que certamente, contribuirá para o aumento de viscosidade do sistema.

\subsection{Avaliação da Turbidez}

A fim de comprovar a quebra do polímero HPG no meio, foram realizados testes de turbidez, onde foi possível avaliar quantitativamente o percentual de polímero no meio, onde a adição de álcool etílico (5 $\mathrm{ml}$ ) foi utilizado para promover a precipitação e assim a turbidez do meio (NTU). A Figura 7 mostra o diagrama de Pareto para se observar a sensibilidade da turbidez em relação aos intervalos dos parâmetros avaliados.

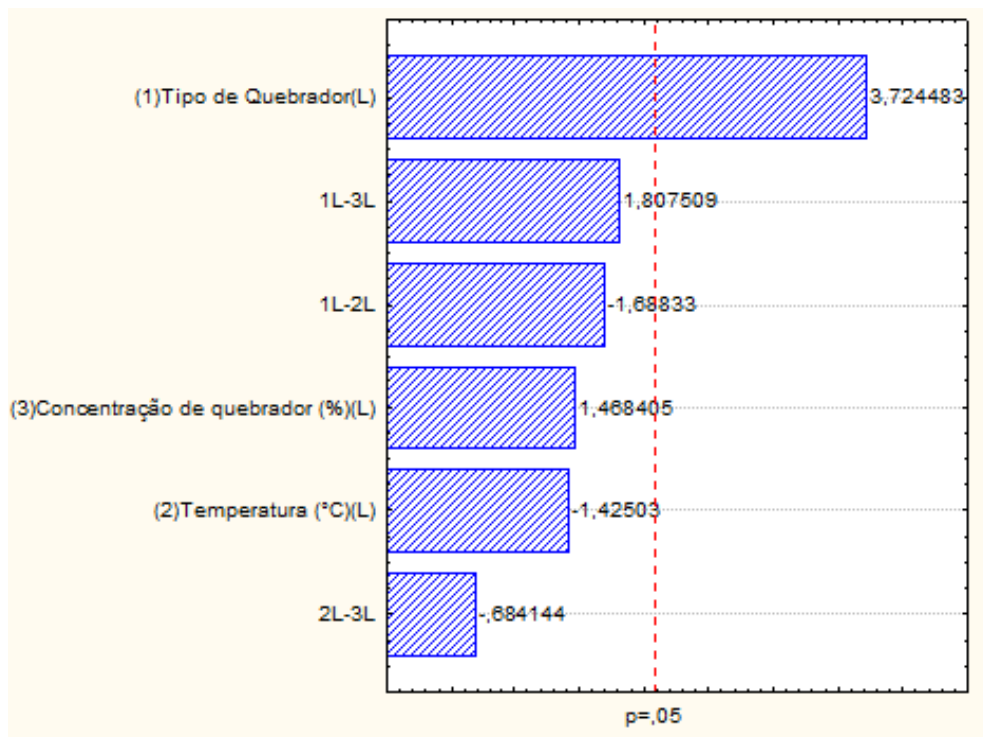

Figura 7: Diagrama de Pareto - Turbidez

De acordo com a Figura 7, o principal efeito estatisticamente mais relevante foi o tipo de quebrador, com sinal positivo, logo espera-se um aumento na turbidez quando o $\mathrm{CaCl}_{2}(+1)$ for utilizado, esse efeito pode ser explicado pela dificuldade deste em degradar o polímero, em virtude da interação com a fração aquosa do gel.

A Figura 8 mostra a turbidez (NTU) em função da concentração de cada quebrador e das diferentes temperaturas. 
(l)

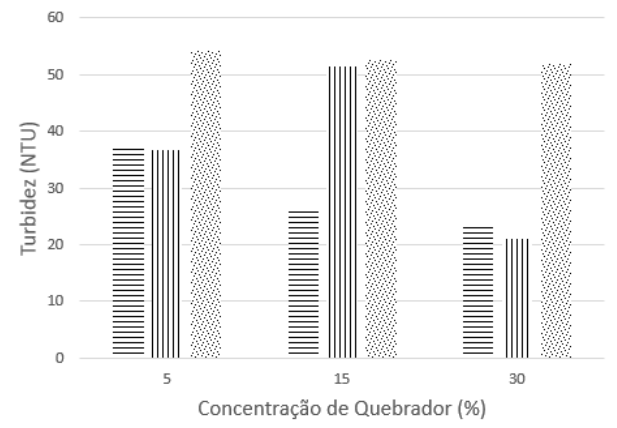

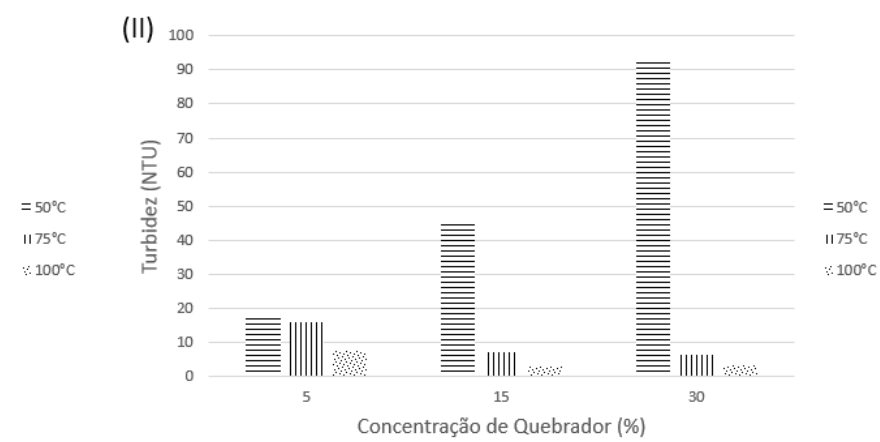

Concentração de Quebrador (\%)

(III)

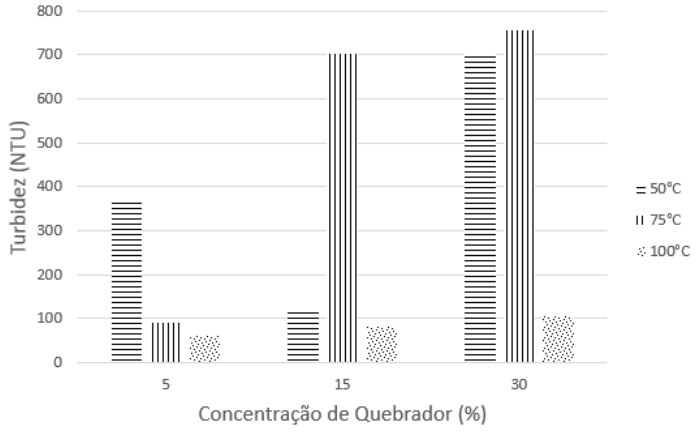

Figura 8: Turbidez (NTU) em função da concentração do quebrador de (I) HCl, (II) persulfato de amônio e (III) cloreto de cálcio

De acordo com a Figura 8 (I), é observada uma alta turbidez no sistema contendo $\mathrm{HCl}$, esse efeito pode ser explicado devido a este apesar de quebrar facilmente os géis em todas as concentrações e temperaturas, deixa uma grande quantidade de polímero insolúvel no meio devido à quebra ocorrer em diferentes pontos da cadeia do polímero, acarretando, portanto, um aumento no dano a formação.

Na Figura 8 (II) percebe-se uma menor turbidez devido a menor quantidade de frações de polímero no meio, esse efeito se deve a presença do ânion persulfato $\left(\mathrm{S}_{2} \mathrm{O}_{8}\right)^{-2}$, visto que este é considerado um agente oxidante muito forte e capaz de degradar uma série de compostos orgânicos e à quebra ocorrer exatamente em pontos específicos da cadeia do HPG, o que certamente favorecerá uma uniforme distribuição de massa molar (LATIMER, 1952).

Em paralelo, o sistema utilizando o cloreto de cálcio (Figura 8 (III)) apresentou um maior percentual de polímero no meio, o que corrobora, portanto, com os dados de viscosidade aparente do gel pós-quebra. Esse quebrador funcionou mais como um agente de precipitação do que propriamente como um quebrador, mostrando que sua eficiência de quebra está associada a temperatura e sua concentração no meio. 


\section{CONCLUSÕES}

- No que diz respeito a interação temperatura e tipo de quebrador as maiores perdas de massa foram observadas com a maior temperatura estudada $\left(100^{\circ} \mathrm{C}\right)$ para os quebradores $\mathrm{HCl}$ e Persulfato de amônia.

- Portanto, podemos afirmar com base em todas as informações contidas no presente trabalho que o $\mathrm{HCl}$ 15\% utilizado como quebrador, apesar de promover a quebra completa do gel acaba gerando alguns transtornos associados ao reservatório, podendo promover obstruções e acarretando um aumento no dano a formação. O cloreto de cálcio funcionou mais como um agente de precipitação do que propriamente como um quebrador. Assim, o persulfato de amônia, devido suas propriedades químicas já citadas foi o quebrador mais eficiente que poderá ser usado no processo de fraturamento hidráulico.

\section{REFERÊNCIAS}

Aqualon Techinical Brochure. (2007). Guar and guar derivatives oil and gas field applications. 2506109, 07.

Barati, R.; Hutchins, R.D.; Friedel, T.; Ayoub, J. A.; Dessinges M. (2009, Maio). Fracture impact of yield stress and fracture-face damage on production with a three-phase 2D model. SPE-111457PA, 24.

Carvalho, E. P. (2011). Uso Do Persulfato de Amônio Para Estabilização Da Polimerização Em Suspensão Do Estireno. (Dissertação de Mestrado, Universidade Federal do Rio de Janeiro, Rio de Janeiro). Recuperado de:

http://objdig.ufrj.br/60/teses/coppe_m/ErnaniPinheiroDeCarvalho.pdf

Economides, M.J. Nolte, K. G. (1989). Reservoir stimulation. Schlumberger Educational Services Houston, Texas, $2^{\circ}$ Edição.

Fracfocus. A. (2016). Historic Perspective. Estados Unidos, Recuperado de: https://fracfocus.org/hydraulic-fracturing-how-it-works/history-hydraulic-fracturing

Garcia, J.E.L.; Paula, J.L.; Fernandes, P.D.; Martins, J.A.S.; Rodrigues,V.F., Ferreira, F.H. (1997). Fraturamento hidráulico. Centro de Desenvolvimento de Recursos Humanos Norte-Nordeste (CENNOR), Petrobrás.

Grothe, V. P. (2002). Estudo da filtração de fluidos reticulados em simulações físicas de faturamento hidráulico. (Dissertação de Mestrado, Universidade Estadual de Campinas, São Paulo). Recuperado de:

http://www.bibliotecadigital.unicamp.br/document/?code=vtls000197607

Gupta, A.; Shah, S.N.; Gadiyar, B. (2000, June). The effect of fracturing fluid leak-off on the productivity of high permeability oil reservoirs. Journal of Canadian Petroleum Technology, 39, (6.), 26.-31. 
King, G. E. (2012). Hydraulic Fracturing 101: What Every Representative, Environmentalist, Regulator, Reporter, Investor, University Researcher, Neighbor and Engineer Should Know About Estimating Frac Risk and Improving Frac Performance in Unconventional Gas and Oil Wells, SPE152596-MS. Recuperado de: https://www.onepetro.org/conference-paper/SPE-152596-MS LATIMER, W.M. (1952). Oxidation states of elements and their potentials in aqueous solutions. Englewood Cliffs, N.J. Prentiee -Hall. Inc.

Liu, D.; Fan, M.; Yao, L.; Zhao, X.; Wang, Y. (2010, September). A new fracturing fluid with combination of single phase microemulsion and gelable polymer system. Journal of Petroleum Science and Engineering, 37, 267-271.

Neta, L. S. F. (2003). Obtenção e estudo das propriedades de um novo fluido (gel) de fraturamento hidráulico biocompatível. (Monografia, Universidade Federal do Rio Grande do Norte). Recuperado de:

http://www.nupeg.ufrn.br/documentos_finais/monografias_de_graduacao/sergina.pdf

Oliveira, A. A. (2012). Seleção de poços para operações de estimulação utilizando inteligência artificial. (Monografia, Universidade Federal do Rio de Janeiro). Recuperado de: http://monografias.poli.ufrj.br/monografias/monopoli10004071.pdf

Pereira, I. R. A., Medeiros, F.B., Fagundes, F.P., Girão, J.H.S., Balaban, R.S. (2011). Efeito da força iônica sobre as propriedades reológicas de géis de fraturamento à base de goma guar e hidroxipropilguar. 60 CONGRESSO BRASILEIRO DE PESQUISA E DESENVOLVIMENTO EM PETRÓLEO E GÁS. Florianópolis, SC. Recuperado de: http://www.portalabpg.org.br/PDPetro/7/publicacoes/repositorio/trabalhos/4605042405201188 0.pdf

Santanna, V. C. (2003). Obtenção e estudo das propriedades de um novo fluido de fraturamento hidráulico biocompatível. (Tese de Doutorado, Universidade Federal do Rio Grande do Norte). Recuperado de:

http://www.nupeg.ufrn.br/downloads/mono_diss_teses/teses_de_doutorado/vanessa.pdf

Silva, J. C., Filho, W.D. C. (2012). Projeto de fraturamento hidráulico em rochas cristalinas para estimulação de poços e recarga artificial na área piloto de samambaia. In: 70 CONGRESSO BRASILEIRO DE ÁGUAS SUBTERRÂNEAS. Bonito: MS. Recuperado de: https://aguassubterraneas.abas.org/asubterraneas/article/view/22816/14976

Stringfellow, W. T., Domen, J. K., Camarillo, M. K., Sandelin, W.L., Borglin, S. (2014). Physical, chemical, and biological characteristics of compounds used in hydraulic fracturing, Journal of Hazardous Materials, (275). 37-54. Recuperado de: http://www.sciencedirect.com/science/article/pii/S0304389414003021

Yew, C. H. (1a Ed) (2008). Mecânica do fraturamento hidráulico. Rio de Janeiro. Editora E-papers.

Vengosh, A., Jackson, R. B., Warner, N., Darrah, T.H., Kondash, A. (2014). A Critical Review of the Risks to Water Resources from Unconventional Shale Gas Development and Hydraulic Fracturing in the United States. Environ. Sci. Technol. 48 (15), 8334-8348. Recuperado de: http://pubs.acs.org/doi/abs/10.1021/es405118y 
Zamora, F., Thompson, J. Kakadjian, S. R., Garza, J.L. (2010). Patente PI 1101503-9 A2, número 12/758, 466. Estados Unidos. 